Running head: SOCIAL CONTEXT INFLUENCES LAUGHTER

Social context influences the acoustic properties of laughter

\author{
Adrienne Wood
}

NOTE: This is a preprint for the following publication:

Wood, A. (2020). Social Context Influences the Acoustic Properties of Laughter. Affective Science. https://doi.org/10.1007/s42761-020-00022-w

Author details. Department of Psychology, University of Virginia, Charlottesville, Virginia, United States. Email: adrienne.wood@virginia.edu 


\begin{abstract}
Laughter serves different functions depending on the social context. Sometimes laughter feels good for producers and listeners, serving as a social reward. Sometimes laughter smooths over social tension, promoting affiliation. And sometimes laughter reprimands or teases the target, establishing dominance. The present study examines whether the acoustic properties of spontaneous laughter reflect the context in which it occurs. Pairs of participants (complete recordings $\mathrm{N}=141$ ) watched and discussed humorous videos associated with the social tasks of reward, affiliation, and dominance. The acoustic profiles of the 3,370 laughs extracted from their conversations depended on the social context. Affiliation laughter was shorter, quieter, and muffled, while dominance laughter was more unpleasant (e.g., brighter, less voiced, noisier). The combined acoustic variables discriminated at greater than chance accuracy between the social contexts. This research accounts for some of the acoustic variability of laughter and highlights the functional flexibility of nonverbal signals.
\end{abstract}

Keywords: laughter, nonverbal behavior, acoustic analysis, humor 


\section{Social context influences the acoustic properties of laughter}

Imagine your coworkers laugh when you tell a joke. They may be laughing at the joke. Or perhaps they are laughing out of politeness. Or maybe they are laughing at you and your pathetic attempt at a joke. How do you know? If laughter simply conveyed genuine or artificial positive affect (Bryant \& Aktipis, 2014; Lavan et al., 2015), then you would have to rely on knowledge about the social context and your coworkers to disambiguate their laughter's meaning (Curran et al., 2018).

But perhaps the acoustic difference of howls, chuckles, and snickers contains meaningful information. We propose laughter serves at least three social tasks (Wood \& Niedenthal, 2018): contagious, pleasant-sounding laughter induces positive affect in the recipient to reward their behavior (Owren \& Bachorowski, 2003); muted, low-arousal laughter that sounds like it comes from a smaller and unthreatening body signals affiliation, reassuring the recipient (Mehu \& Dunbar, 2008); and unpleasant laughter that makes the producer sound larger enforces norms and conveys dominance (Szameitat et al., 2009). Of course, context adds meaning to a signal (Curran et al., 2018), so laughter acoustics should be more distinctive and contain more information in ambiguous contexts (Wood \& Niedenthal, 2018). Multiple contextual and acoustic meanings might even enable a laugh to accomplish multiple tasks simultaneously (Scarantino, 2018). For instance, laughing friends can affiliate with each other by conveying shared dominance over another person.

In a recent study, participants' judgments of how rewarding, affiliative, or dominant a laugh sounded depended on a number of acoustic properties (Wood et al., 2017). This work suggested perceivers of laughter infer social meaning (beyond just whether the laugh is authentic or volitional) but does not tell us whether people naturally produce different forms of laughter 
depending on the social context.

The present work examines the acoustic form of natural laughter in ecologically valid social contexts. Dyads watched, discussed, and spontaneously laughed about humorous videos relevant to reward, affiliation, and dominance. Prior work could not separate individual differences in laughter (e.g., people with smaller vocal tracts sound more affiliative) from withinperson variability (Wood et al., 2017). The present work identifies modifiable properties of laughter that potentially convey an individual's state. And unlike prior work that studied laughter across relationship types (e.g., friends and strangers; Bryant et al., 2016; Oveis et al., 2016), context was manipulated within-dyad, isolating context effects from relationship effects. Our findings suggest laughter is not a homogenous expression of positive affect; rather, laughter conveys nuanced social messages (Scarantino, 2018).

\section{Method}

We report all data exclusions, all manipulations, and all measures. Materials, participant laughter clips, humorous video stimuli, data, and analyses are available online (https://osf.io/a8t29/). We used Qualtrics software (Qualtrics, Provo, UT) for all video presentation and rating tasks. The study was approved by the University of Wisconsin-Madison I.R.B. and all participants gave their informed consent prior to participation.

\section{Participants}

Pairs of undergraduate Introduction to Psychology students at the University of Wisconsin-Madison participated in exchange for extra credit. To maximize lab efficiency, we over-recruited for each timeslot, and when three participants arrived one was randomly assigned to complete a separate video stimulus rating task (described in Appendix D); when only one participant attended a session, they too completed the video stimulus rating task. 82 dyads (164 
participants) participated in the main task (94 female, 67 male, 1 nonbinary ${ }^{1} ; M_{a g e}=18.82$,

$S D_{a g e}=0.74 ; 20$ Hispanic/Latinx, 139 non-Hispanic/Latinx, 4 did not report; 1 American

Indian/Alaskan Native, 17 Asian, 1 Asian and Native Hawaiian/Pacific Islander, 2 Black/African

American, 1 Native Hawaiian/Pacific Islander, 120 White, 2 White and American Indian/Alaska

Native, 3 White and Asian, 3 White and Black/African American, 2 White and Black/African

American and American Indian/Alaska Native, 5 "other", 5 not reporting). Of the 82 original

dyads, 8 experienced computer program failure that resulted in the dyad not being able to

complete the task. For 7 dyads, 1 of the participants experienced audio equipment failure, so we

analyzed the laughter data from their partners. This resulted in audio data from 67 complete

dyads and 7 single participants whose partners' audio recordings were lost $(\mathrm{N}=141)$. We had no

expected effect size to guide a priori power analyses; instead, the sample size was determined

based on how many participants signed up within a semester, with the goal of exceeding 100

subjects given the within-subject nature of the manipulation. A post-hoc power analysis using the

pwr library in R (Champely, 2020) indicated that with our sample size and within-subject design, we would be able to detect even a small effect (Cohen's $d>=0.24$ ) with $80 \%$ power.

\section{Video stimuli}

Stimuli were 120 brief humorous videos (ranging in length from 1-30 seconds) that research assistants agreed elicited responses associated with the social functions of reward, affiliation, and dominance (40 per social function). 50 videos came from an existing emotioneliciting database (Cowen \& Keltner, 2017) and 70 were found on YouTube. Importantly, videos

\footnotetext{
${ }^{1}$ The person identifying as gender nonbinary signed up for the female version of the study and worked with a female partner. They also had to identify as female in the Intro to Psych mass survey in order to qualify for the female version of the current study. Since all of my analyses include gender as a moderator or covariate, we could either exclude this person from the analyses or put them in the female category. For purely statistical reasons we elected to include their data and coded them as female, but we acknowledge the issues with dichotomizing gender.
} 
were included only if they made sense and were humorous without audio, because sound would interfere with the audio recording of participants.

For the social function of reward, we selected videos perceived by research assistants to be highly humorous in a straightforward sense. For affiliation, we selected videos that were humorous but also elicited responses associated with tenderness, care, empathy, in-group signaling, or cuteness. Affiliative laughter is theoretically meant to soothe, ameliorate, and signal nonthreat or acknowledgment, so videos were selected with these tasks in mind. Finally, the videos selected for the social function of dominance were those that elicited responses of derision, ridicule, wanting to put the person/people "in their place", or feeling that someone "got what they deserved." Four research assistants watched the videos and selected the social task they considered most relevant; videos without the agreement of at least three raters were replaced.

The research team assigned 40 videos to each social context (Reward, Affiliation, Dominance) based on the proposed social tasks and prior research on humor (for details, see Wood \& Niedenthal, 2018). Independent ratings from naïve participants were not obtained beforehand, but ratings from participants who completed the Stimulus Rating Task instead of the primary tasks $(\mathrm{N}=118)$ are reported in Appendix D. Stimulus Rating Task participants reported feeling the most derision towards and feeling "better than" the targets in the Dominance videos and they felt the most tenderness towards the targets in the Affiliation videos, but the Reward videos were not rated consistently higher than the other videos on any of the other judgments. For instance, because reward involves reinforcing social bonds, we expected Reward videos be rated highest on the following item: "I want to spend time with the person/people in this video". Although the pre-assignment of videos to the social contexts turned out to be imperfect, the 
Video Selection Task procedure included an internal validation that ensured the videos used in the final Video Sharing Task were indeed perceived by those participants to fit the intended social context, as we describe below. Participants discussed (and laughed about) only videos that they personally felt fit each function. This is a key feature of the study design because humor styles are idiosyncratic (Cann et al., 2009).

\section{Procedure}

After completing an ice-breaker activity, same-gender pairs of participants independently completed a Video Selection task in which they watched a series of brief humorous videos preselected for their relevance to the social tasks of reward, affiliation, and dominance. Each participant indicated the videos that made them feel states associated with the three social tasks. Their selections then served as the stimuli in the Video Sharing and Discussion task, during which partners watched and discussed each other's selections (see Appendix A for overview of procedure). We recorded the audio of the free-form conversations and extracted all instances of audible laughter.

The study design maximized the social functional relevance of the videos discussed by the dyads. The Video Selection task ensured that the participants actually interpreted the videos in the way we intended and eliminated the need for a separate manipulation check. The Video Selection phase was also developed to maximize laughter: participants are potentially more likely to laugh to signal a rewarding, affiliative, or dominant response to a humorous video if they know their partner chose the video for those tasks. They might accomplish the metaconversational goal of affiliating with their partner by conveying these other social messages towards the video targets (Scarantino, 2018). And finally, we chose to record and analyze laughter elicited during the social Video Sharing and Discussion task because laughter is a social 
behavior that is much less frequent when people are alone (Devereux \& Ginsburg, 2001).

Ice-breaker activity. Upon arrival to the lab and after being assigned to the partner task, participants were told the study was about "how people understand and talk about humor." Participants gave their informed consent and were left alone in the lab room to spend 7 minutes on the ice-breaker activity. This task was designed to relax the participants and get them familiar with one another to maximize the likelihood of laughter in the final phase of the study. Each participant received a sheet of paper with a series of questions that they took turns asking each other. They began by saying their names, intended majors, and hometowns, then answered a series of light-hearted get-to-know-you questions (18 were provided but pairs rarely completed them all in the 7 minutes; example questions: "If you had a theme song that played whenever you walked into a room full of people, what would it be?"; "What would be the worst 'buy one get one free' sale of all time?"). The experimenter returned after 7 minutes to move the participants on to the next phase of the study.

Video selection task. Participants moved to their separate workspaces (see Figure 1) and the experimenter read the instructions out loud while participants followed along on their computers. Instructions explained that they would be "watching 3 playlists that each contain 20 very short videos....In each playlist, you'll be asked to pick at least 5 videos (out of 20) that fit a description....The description changes for each playlist, so read the instructions carefully....If, by the end of the playlist, you haven't picked at least 5 [videos], you can go back and change some of your answers."

Participants then privately viewed three playlists of 20 silent videos (Reward, Affiliation, and Dominance). Each participant in a dyad saw a unique playlist of videos so they could later show their selected videos to their partners without their partners having already seen them. The 
order of the Reward, Affiliation, and Dominance playlists was randomized across participants, as was the order of the videos within each playlist. Participants then picked at least five videos per playlist that elicited feelings associated with the intended social function. Specifically, after watching each video, they responded "true" or "false" to one of the following prompts (depending on the playlist):

- I feel happiness, amusement, or joy towards the person/object in this video. (Reward)

- I feel warmth, friendliness, or care towards the person/object in this video. (Affiliation)

- I feel derision, disdain, or mocking towards the person/object in this video. (Dominance)

Figure 1. Lab setup for Video Sharing and Discussion Task.

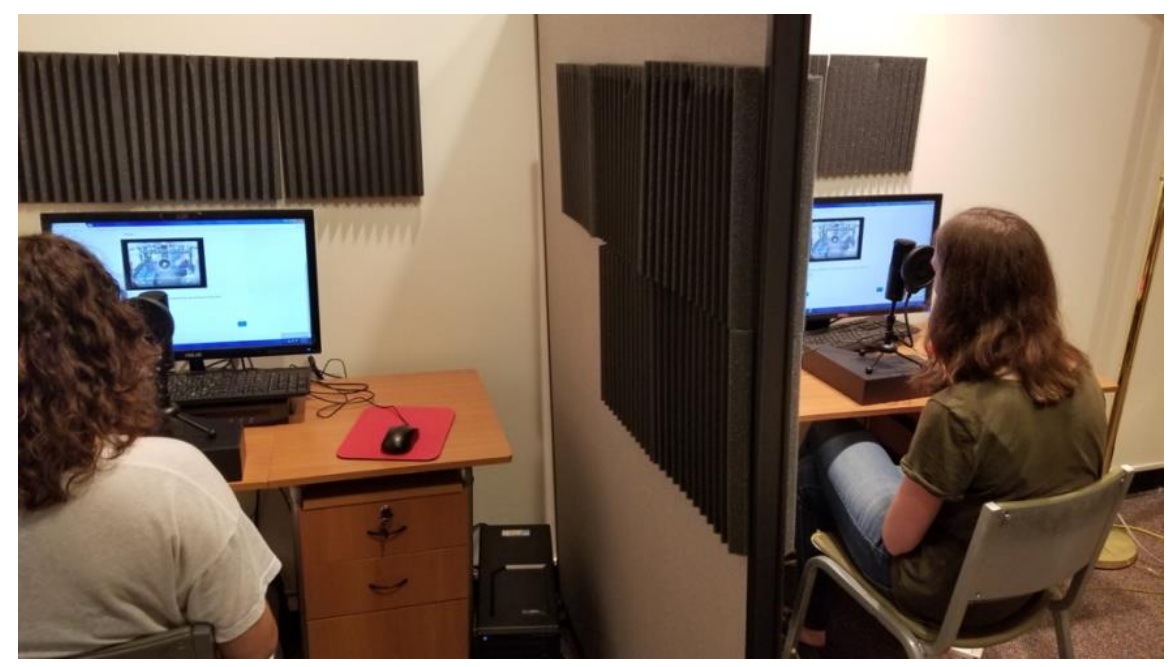

Footnotes. After the Ice-Breaker Activity, participants worked at separate stations with monitors and microphones and were visually separated but could easily hear one another.

The forced-choice true/false response was preferable to a Likert-type response because it categorically identified videos that could be used in the next task. The task was not complete until they had responded "true" to at least 5 videos per playlist, which was required for the subsequent Video Sharing task. Participants responded "true" to about half of the videos (about 
10 per playlist, suggesting they had no trouble finding at least 5 videos that elicited the intended feeling (see Table S2 for descriptive statistics of participants' responses in the Video Selection phase). The experimenter remained in the lab room during this task, which typically took about 15 minutes to complete.

Video sharing and discussion task. After both participants finished the Video Selection task, the experimenter used a KVM switch to set both participants' monitors to display and control the same computer. This allowed participants to view stimuli simultaneously while being separated by the partition wall. Not only did the partition improve the quality and separation of the two microphones' recordings, but it also removed participants' ability to communicate with visual nonverbal signals, presumably requiring them to convey more with their voices (Andelic et al., 2019). The experimenter moved each participant's microphone in front of them and asked them to adjust their posture so they could speak directly into the microphones (see next section for details on audio recording). They then read the instructions for the Video Sharing and Discussion task: "Now that both of you have picked your videos, you will watch them together and discuss why you picked them. Remember that we are interested in how people talk about humorous things, so talk about whatever aspect of the video you want....Before you play each video, the partner who picked the video will be prompted to....explain what the video is about and why you thought it fit that particular description." Before viewing and discussing each video, the participant who originally selected the video was prompted to clearly state the video's identification number into the microphone. After reading the instructions out loud and answering any questions, the experimenter began recording the audio from both mics and left the room so the participants could complete the task in private.

The Qualtrics survey—simultaneously seen by both participants on their own monitors- 
presented a total of 30 videos that the participants selected in the previous task: 5 videos per social function per participant. If participants selected more than 5 videos in the Video Selection task, the software chose 5 of them randomly. The order of the social contexts was randomized across dyads, and within a social context block, the order of the two participants was counterbalanced. For instance, Participant A might be prompted first to play and explain her 5 happiness/amusement/joy videos (in random order), then Participant B would play and explain her 5 happiness/amusement/joy videos, then they would move on to the next social context. This task took approximately 15 minutes to complete. Participants then completed a brief demographics survey and were debriefed and thanked for their participation.

\section{Lab setup and audio equipment}

The sessions took place in a small, carpeted lab room with sound absorbing foam tacked to the walls near the participants' two desk stations (see Figure 1). A 66Hx48W" fabric-covered partition separated the participants' stations, over which the participants could easily hear each other at a conversational level of speaking. Audio was captured with 1ByOne USB microphones (44.1 kHz sampling rate) set to cardioid pattern mode so as to capture a more focused sound stream from the participant next to the mic. A 4" Zramo pop filter was placed between the mics and the participants' mouths to reduce popping sounds during speech and to protect the mics. The mics were mounted on small desktop tripods set on a 6" tall box, which positioned them at a comfortable height for a person of average height. Audio was recorded separately on each participant's computer using Audacity (Version 2.2.1), with the gain set to $10 \%$, and saved as a WAV file. Despite our best attempts to isolate each participant's voice, the distant participant can be heard on the near participant's recording, albeit at orders of magnitude lower amplitudes.

\section{Laughter extraction and acoustic property characterization}


Seven trained undergraduate research assistants and the author isolated all instances of laughter or speech-laughter from the Video Sharing and Discussion recordings. Some laughter occurred while the dyads were watching the videos and some laughter occurred while they discussed the videos. To avoid biasing the results, we included all laughs, regardless of the surrounding conversational content. If anything, this liberal definition of "reward," "affiliative," and "dominance context" made us less likely to find significant acoustic differences across contexts, because dyads' conversations occasionally wandered, causing them to laugh for other reasons.

We defined laughter as the audible forced expulsion of air through the vocal cords, which may be unvoiced or voiced. If voiced, it is heard as a vowel sound occasionally interrupted by a glottal stop (e.g. the pause of airflow in the middle of the expression "uh-oh") or a glottal fricative consonant (/h/; Nwokah, Hsu, Davies, \& Fogel, 1999). We included multi-burst ("ha ha ha") and single-burst ("ha") instances of laughter in the current definition. Five separate research assistants then listened to the isolated laughter samples (each laugh was rated by three people) and indicated whether they were pure laughter or speech-laughter. Speech-laughter is speech colored with the breathing patterns and prosodic markers of laughter, existing somewhere between normal spoken word and pure laughter both physiologically and acoustically (Nwokah et al., 1999). Laughter bursts that immediately preceded, interrupted, or followed speech were coded as laughter; only laughter that occurred during a recognizable word or phrase was coded as speech-laughter. We extracted 4,713 laughter samples in total, 1,343 of which were coded as speech-laughter. We included all laughs in the count analyses but used only the 3,370 nonspeech laughs in the acoustic analyses.

We characterized the acoustics of the non-speech laughs with the PraatR (Albin, 2014) and 
soundgen (Anikin, 2018) libraries in R (see Online Materials for complete code). We measured 21 acoustic properties, including 11 used in previous work (Wood et al., 2017). Although the present study complements the prior perceiver-based work, we approached the acoustic analyses from an exploratory perspective (thus the addition of 10 more acoustic measures, which capture additional properties in the feature space). We did not know which, if any, of the acoustic properties identified in our prior work reflected properties that producers modify across social contexts and laughter instances, as opposed to fixed properties of their voices that make their laughter sound generally more rewarding, affiliative, or dominant. And indeed, many acoustic features identified in the prior perceiver-based work — in particular, pitch-based variables_-did not vary across contexts in the present work.

We log-transformed variables when necessary to correct for positive skew. See Table $\mathbf{S 4}$ for descriptive statistics of each variable. In the following list, SG indicates variables measured with soundgen and $\mathrm{P}$ indicates variables measured with Praat.

1. Duration (SG): Length of the sample in seconds (log-transformed).

2. Number of bursts (SG): The number of sound bursts, or laughter beats ("ha"), detected in the sample. The segment function in the soundgen package identifies local maxima in the amplitude envelope. The detection threshold that defined a burst or syllable was set to $70 \%$ of the global mean amplitude, and the shortest detectable syllable was set to $40 \mathrm{~ms}$.

3. Interburst interval mean (SG): The average time between detected bursts (logtransformed) — in other words, the laughter tempo — with a shorter interval indicating more rapid laughter bursts.

4. Amplitude mean (P): The mean loudness in decibels (log-transformed).

5. Amplitude SD (SG): Variability in loudness over the course of the sample (log- 
transformed).

6. F0 mean (P): Mean fundamental frequency, or pitch, as calculated using Praat's autocorrelation technique, converted to semitones. Not all laugh samples have a detectable pitch (resulting in missing data for subsequent F0 variables). Praat's pitch detection technique is not sufficiently conservative, as it detected a pitch on $96.3 \%$ of the samples, many more of which are audibly unvoiced and should therefore have no detectable pitch.

7. F0 mean (SG): Soundgen's estimate of pitch, which integrates and processes pitch estimates using four non-independent algorithms: autocorrelation, lowest dominant frequency, cepstrum, and spectrum. Soundgen detected a pitch on only $27.4 \%$ of laugh samples, so we report this more stringent estimate (converted to semitones) alongside that of Praat's more error-prone pitch detector. Pitch was not detected at different rates across the Social Contexts.

8. Fo SD (SG): The within-laugh standard deviation of Soundgen-detected pitch, logtransformed. This represents the pitch variability across an entire laugh sample.

9. F0 range $(\mathrm{P})$ : The difference between the lowest and highest F0 in the sample, in semitones.

10. F0 slope $(\mathrm{P})$ : Mean absolute F0 slope in semitones, which measures how sharply the pitch changes occur. Calculated by dividing the difference between a local F0 maximum and minimum (at intervals of .01 seconds) by the duration it takes to go from one to the other.

11. F0 variability $(\mathrm{P})$ : Moment-to-moment standard deviation of $\mathrm{F} 0$ divided by the total duration of the sample, log-transformed; captures the average variability in pitch. Note how this differs from F0 SD, above: a vocalization could have high a F0 SD and low F0 
Variability if, for instance, it gradually went from a high to low pitch.

12. F1 mean $(P)$ : The first peak in the sound spectrum above $F 0$, which helps determine the vowel sound of a vocalization. Transformed to semitones.

13. F2 mean $(\mathrm{P})$ : The second peak in the sound spectrum, above F1, which helps determine the vowel sound of a vocalization. Transformed to semitones.

14. F2-F1 (P): The distance between F2 and F1. As the difference increases, the vowel sounds higher and the vocal tract sounds relatively smaller. Transformed to semitones.

15. Portion voiced $(\mathrm{P})$ : The portion of frames that are voiced as opposed to unvoiced. Voiced segments are nearly periodic, while unvoiced segments are noisier.

16. Harmonics-to-noise ratio $(\mathrm{P})$ : The average degree of periodicity in $\mathrm{dB}$; a higher value indicates a purer, more tonal sound, and a lower value indicates a noisier vocalization.

17. Spectral center of gravity $(\mathrm{P})$ : The "center of mass" of the vocalization, logtransformed. Also known as the spectral centroid. Higher centroids sound brighter and/or more nasal.

18. Spectral slope mean (SG): The slope of the linear regression fit to the spectrum-in other words, how quickly the energy in the sound tails off in the higher frequencies (for all frequencies below $6000 \mathrm{~Hz}$ ). This will be a negative slope, with less energy in the higher frequency ranges, but a steeper slope with a faster drop off will sound mellow and muffled, while more power in the higher harmonics (a more gradual drop off of energy) will sound thinner and brighter.

19. Entropy mean (SG): The average Weiner entropy of the spectrum, which serves as another estimate of noise. As this number approaches 0 , the vocalization sounds more tonal, and as it approaches 1, it becomes white noise. 
20. Entropy SD (SG): The standard deviation of the Weiner entropy of the spectrum across the laugh sample. A higher value indicates fluctuations in entropy over time, with the voice varying in its noisiness.

21. Modulation spectrum roughness ( $\mathrm{SG}$ ): The modulation spectrum can be thought of as a two-dimensional Fourier transform that captures spectro-temporal (rather than just spectral) patterns in a vocalization. Soundgen calculates the percent of energy in the temporal "roughness range," which we set to the default of $30-150 \mathrm{~Hz}$, with a Fourier transform window of $40 \mathrm{~ms}$ and a step size of $10 \mathrm{~ms}$. The roughest laughs were unvoiced pants or snorts, while the least rough laughs were closed-mouth, voiced hums.

\section{Results}

We begin by reporting differences in laughter frequency across the social contexts. We then used multinomial logistic regression to predict social functional context from all acoustic variables. This approach indicates whether the combined acoustics distinguish between reward, affiliation, and dominance-context laughs.

Finally, in a reverse approach, we report analyses predicting each of the 21 acoustic properties using dummy-coded social context and dyad gender. This indicated which acoustic properties systematically varied across the social contexts. In these models we controlled for the video stimuli's average funniness ratings (as judged by the separate sample of participants who completed the video stimulus rating task described in Appendix D). We did not want any incidental differences in funniness across the three video contexts to confound the effects of the social context manipulation on acoustics. The conclusions from these models are largely unchanged when we do not control for the funniness of each video (see Appendix $\mathbf{C}$ for those results). Two marginal effects of social context on acoustics became significant when we no 
longer controlled funniness; we explain these differences below. Given the exploratory nature of these analyses, which include 21 separate dependent variables and multiple re-levelings of categorical predictor variables, we controlled the false-discovery rate rather than the family-wise error rate (Benjamini \& Hochberg, 1995). This approach specifically controls false positives, as opposed to general error.

For all analyses using acoustic variables, we excluded laughter instances coded as speech-laughter, leaving 3,370 non-speech laughs (M=23.90 per participant). See Appendix B for additional analyses, including the extent to which partners influenced each other's laughter rate.

\section{Rate of laughter across the social contexts}

We first analyzed the frequency with which participants laughed as a function of gender and Social Functional Context. Using a generalized linear mixed-effects model with a Poisson distribution, we regressed Laugh Count on a unit-weighted, centered Gender variable (male = .5 , female $=.5$ ), dummy variables comparing the 3 Social Functional Contexts ${ }^{2}$, and the interaction between Gender and the dummy variables. We re-ran this model 3 times with each of the 3 Social Functional Contexts as the reference level for the dummy variables. Subjects were fully nested within dyads, allowing for by-subject/dyad random intercepts.

Complete model coefficients are reported in Table S3, along with unadjusted p values and Benjamini-Hochberg adjusted p values. Controlling for all other variables and averaged between males and females, participants laughed more in the reward than affiliation context, $b=$ $0.185, S E=0.036, z=5.14, a d j . p<.001$. Participants also laughed more in reward than

\footnotetext{
2 Models using a dummy variable approach failed to converge even when we took steps to simplify the random effects structure. We chose not to unnest participants from dyads, which probably would have allowed the dummy variable models to converge, since within-dyad laughter frequency is moderately correlated (see next section of analyses).
} 
dominance video contexts, $b=-0.126, S E=0.035, z=-3.56, a d j . p=.002$; see Figure S2). There was not a significant difference in the laughter rate in the Affiliative and Dominant contexts.

\section{Estimating social context from combined acoustics}

Next, to determine whether the combined acoustic variables could discriminate between the social contexts, we used multinomial logistic regression with the nnet library for R (Venables \& Ripley, 2002). We excluded the F2-F1 acoustic variable because it was highly collinear with F1 Mean. We compared two nested models that predicted three-category Social Context as the outcome: one model included the 20 acoustic variables and Gender as predictors, and the second model had only Gender as a predictor. This multiple-degrees-of-freedom comparison estimated how well the combined acoustic variables could discriminate between Social Contexts, controlling for Gender. Such a nested model comparison approach requires complete observations, so we handled missing acoustic data using multiple imputation with the mice $\mathrm{R}$ function (Buuren \& Groothuis-Oudshoorn, 2011). Because the imputed data and nested model comparison could not be combined with a mixed-effect modeling approach, we first adjusted for between-participant differences in laughter by centering the acoustic variables within-participant. Centering the variables around each participant's mean removed the sizeable between-participant variability in acoustics (van de Pol \& Wright, 2009).

The likelihood ratio test indicated that the full model performed significantly better in predicting Social Context compared to the gender-only model, $L R T=119.736, p<.0001$. A Pearson's Chi-squared test comparing the laughs' model-predicted Social Context to their true Social Context further suggested the acoustic variables discriminate between the contexts at better than chance, $\chi^{2}=73.62, p<.0001$. Across the three contexts, $41.4 \%$ of laughs were accurately categorized, which is significantly better accuracy than the No Information Rate, 
$36.8 \%, p<.0001$ (see confusion matrix, Table S1, and online materials for diagnostics for each Social Context). We repeated the analysis using only the 603 laughs for which soundgen's more conservative pitch estimator was able to detect a pitch; the conclusions were identical using this much smaller laughter set; LRT $=65.47, p=.007, \chi^{2}=40.71, p<.0001$ (see online materials for complete statistics).

As illustrated in Table S1, of the laughs predicted to be from a given context, the majority were indeed produced in that context. However, the model is biased towards categorizing laughs as rewarding: laughs from all 3 contexts were most likely to be categorized as Reward laughs. This bias in the model may partly result from an overrepresentation of Reward context laughs in the sample. As mentioned earlier, participants laughed significantly more in the Reward context (38\% of the total laughs in the acoustic analyses) compared to the Affiliation (31\%) and Dominance (31\%) contexts. It may be the case that participants produced reward laughter in the Affiliative and Dominant contexts, which would be unsurprising given how unconstrained the conversations were and the likely overlap of social tasks in any one context.

To summarize, the 20 acoustic properties can be used to categorize the laughs according to context at greater than chance accuracy. Even so, there is a substantial amount of ambiguity and overlap in the acoustics. To the extent that the recipients of the laughs still understood them to be conveying reward, affiliation, or dominance, they may have been relying on contextual cues rather than features of the acoustic signal (Curran et al., 2018).

\section{Effect of social context on acoustic properties}

To better understand the bivariate relationships between the acoustics and social context, we ran a series of 21 models regressing acoustic variables (see Table $\mathbf{S 4}$ for descriptive statistics) on dummy-coded Social Context. We included as predictors unit-weighted and 
centered Gender $($ male $=-.5$, female $=.5)$ and the interaction between Social Context and Gender, with by-subject and by-stimulus random intercepts.

We also included a stimulus-level covariate to rule out an alternate interpretation of the present findings. An alternative to the current hypothesis - that the acoustics of laughter reflect specific social functions_-posits that a large component of variability in laughter acoustics is due to the intensity of laughter and does not convey additional social information (Curran et al., 2018). We therefore controlled for the average Funniness ratings for each video stimulus, based on the judgments of a separate set of 118 participants (see Appendix D for rating study details). We reasoned that laughter will generally be more "intense" in response to funnier stimuli, so controlling for the funniness of the videos allows us to examine effects of Social Context on laughter acoustics above and beyond any intensity effects.

We first ran 21 separate regressions with Reward as the reference level of Social Context, which provided estimates of Reward vs Dominance and Reward vs Affiliation. We then recoded Social Context with Affiliation as the reference level, from which we obtained the Affiliation vs Dominance contrast (Decomposing, Probing, and Plotting Interactions in $R$ ). We report the significant main effects of Social Context (averaged over gender), significant main effects of Gender, significant interaction effects, and significant effects of video Funniness. Complete estimates for the Social Context main effects are reported in Figure $\mathbf{2}$ and all model estimates are available in table format in online materials. Analyses without the Funniness covariate are reported in Appendix C.

Main effects of social context. The significant effects of Social Context on acoustics (after controlling the false-positive rate) are summarized next. We will refer to laughs as "reward", "affiliation," and "dominance" laughs, but strictly speaking, they are the laughs that 
occurred during the reward, affiliation, and dominance video-viewing contexts. BenjaminiHochberg adjusted $\mathrm{p}$ values are reported but see Figure 2 for complete statistics.

Reward laughs were longer $(a d j . p<.001)$, had more bursts $(\operatorname{adj} . p=.021)$, and were louder (adj. $p=.016)$ than affiliation laughs. Dominance laughs had less voicing $(a d j . p=.004)$, lower harmonics-to-noise ratios $(a d j . p<.001)$, higher spectral centers of gravity $(a d j . p=.002)$, greater entropy $(a d j . p=.009)$, and more modulation spectrum roughness $(a d j . p=.014)$ than affiliation laughs. Dominance laughs also had lower harmonics-to-noise ratios than reward laughs (adj. $p=.029)$ And affiliation laughs had more negative spectral slopes than reward (adj. $p=.036)$ and dominance (adj. $p=.040)$ laughs.

In the alternate models that did not control for the funniness of the video stimuli (see Appendix C), the marginal effect of Social Context on loudness variability (Amplitude SD) became significant, with reward (adj. $p=.025)$ and dominance laughs (adj. $p=.022)$ having more variation in loudness than affiliation laughs. Dominance laughs also have significantly higher spectral centers of gravity compared to reward laughs $(a d j . p=.048)$ when we did not control for funniness. 
Figure 2. Estimated effect of context on acoustics.

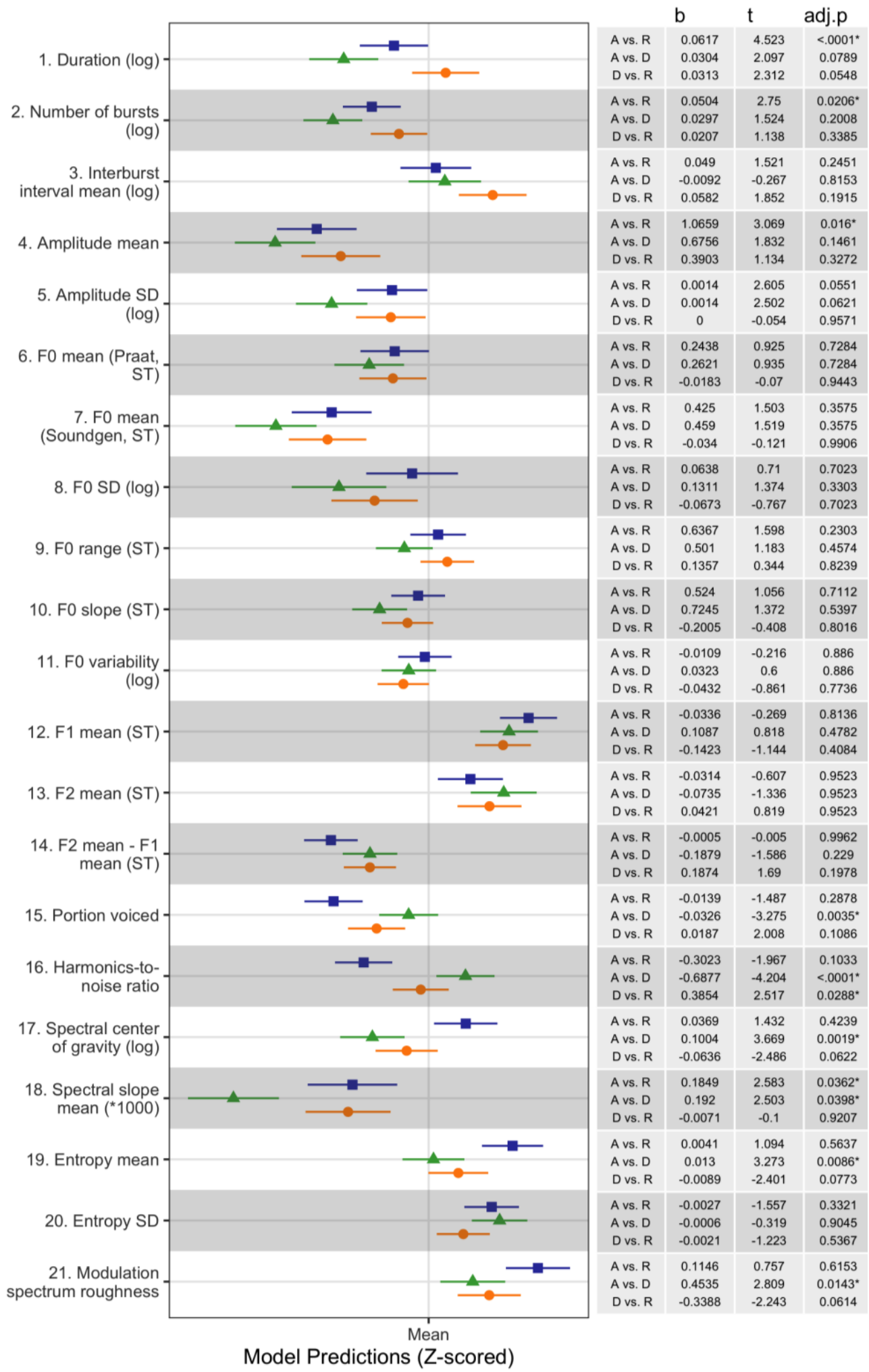

Social Context $\phi$ reward $\uparrow$ affiliation dominance 
Notes. Points indicate model-estimated effects of Social Context on each acoustic variable for a hypothetical gender neutral participant (e.g., when gender is centered between male and female), controlling for the average funniness rating of videos. Model estimates and standard errors of the estimates have been Z-scored to make comparisons across acoustic variables easier. To the right of the graph are the results from the dummy-coded contrasts comparing Reward (R), Affiliation (A), and Dominance (D).

In summary, reward laughs were longer and louder; dominance laughs were noisier (harmonics-to-noise, portion voiced), chaotic (roughness and entropy), and unpleasant (spectral center of gravity); and affiliation laughter was more muffled (spectral slope). These differences cannot be explained by differences in amusement across Social Contexts because we controlled for the funniness of the video stimuli.

Effects of funniness. Controlling for Gender and Social Context and after applying Benjamini-Hochberg adjustments to p-values, the average Funniness ratings of the video stimuli positively predicted the duration of laughter, $b=.044, S E=.009, t(140.24)=4.98, a d j . p<.001$, and correspondingly, the number of bursts, $b=0.035, S E=0.012, t(123.33)=2.85, a d j . p=$ .016. See Table S5 for complete Funniness model estimates. This indirectly validates the use of funniness ratings as a proxy for "intensity," because we might expect a more intense emotional reaction to a stimulus to result in a longer vocalization (keeping in mind that what is intensely funny to a particular conversation dyad may not be intensely funny to the average Stimulus Rating Task participant). Notably, Funniness predicted no other acoustic variables when controlling for Social Context.

Simple effects of gender. See Table S6 for model estimated gender differences in the three social contexts for the 21 acoustic variables. To summarize, compared to male participants, female participants had higher F0 means, lower F1 means, and larger F2 - F1, all characteristic of sexually dimorphic vocal tracts. Females had more laughter bursts in the affiliation context 
than males. Males had greater pitch range and entropy variability than females in their reward laughter. Female laughter fell on the more pleasant end of a number of dimensions compared to male laughter: females had more voicing and harmonics, and reduced roughness. However, males had steeper spectral slopes, indicative of a warmer, more muffled tone.

Interactions between social context and gender. Only 2 social context $\mathrm{X}$ gender interactions were significant after adjusting $\mathrm{p}$ values to control the false positive rate; for those interactions, we examined the simple effects of social context for males and females separately to unpack the interaction terms.

The Reward vs. Affiliation comparison for number of laughter bursts was significantly moderated by Gender, $b=0.088, S E=0.035, t(2921.36)=2.55, a d j . p=.023$. We re-centered the Gender variable over males, then females, to examine the effect of Social Context on interburst intervals separately for each gender. Female affiliation and reward laughter did not differ in the number of bursts, $b=0.006, S E=0.022, t(178.57)=-0.28$, adj. $p=.809$. Males, on the other hand, had significantly more laughter bursts in the reward compared to affiliation context, $b=0.095, S E=0.028, t(408.89)=3.41, a d j . p=.004$.

The Reward vs. Affiliation comparison for pitch standard deviation was also significantly moderated by Gender, $b=-0.447, S E=0.174, t(745.43)=-2.57$, adj. $p=.034$. Female laughter had a higher pitch standard deviation in the reward compared to affiliation context, $b=0.287, S E$ $=0.105, t(94.31)=2.75, a d j . p=.034$. Male reward and affiliation laughter did not differ in pitch standard deviation, $b=-0.160, S E=0.143, t(292.127)=-1.12, a d j . p=.476$.

To summarize the social context $\mathrm{X}$ gender interactions, females but not males had reduced pitch variability — a vocal indicator of arousal — in the affiliation context (compared to 
reward). Males, meanwhile, had fewer bursts in their affiliation laughter (compared to reward), but females did not.

\section{Discussion}

We analyzed the acoustic properties of laughter during conversations about humorous videos relevant to the social tasks of reward, affiliation, and dominance. Affiliation laughter was acoustically "smaller" and muted, fitting with the proposed task of nonthreateningly reassuring recipients. Dominance laughter was unpleasant, noisy, and unpredictable, fitting with the proposed task of punishment. Reward laughter arousing and salient like dominance laughter but lacked its unpleasant-sounding properties.

Other work examining acoustic variability in natural laughter either focused on the differences between spontaneous and volitional laughter (e.g., Bryant et al., 2016), did not manipulate social context but instead used raters to identify categories of laughter (Tanaka \& Campbell, 2011), used pre-existing social relationships as proxies of social functions (Oveis et al., 2016), or only examined laughter rates (Mehu \& Dunbar, 2008).

This is the first naturalistic test of the social functional account of laughter and smiling. Prior work used perceiver ratings of posed expressions (Rychlowska et al., 2017; Wood et al., 2017). The acoustic properties distinguishing the three social contexts here are different from the variables that guided perceiver's judgments about laughter's meaning in prior work. For instance, pitch-based variables guided perceiver judgments (Wood et al., 2017), but here pitch did not vary across contexts. Perhaps the humorous videos used here did not fully capture the proposed social functions of laughter as described to perceivers in prior work. Or perhaps pitchbased features of laughter vary more between-producers than within-producers, and the perceiver-based work confounded trait- and state-level acoustic features. 
This work lays the groundwork for developing a behavioral coding system for inferring the social intentions of laughter from acoustic data. Future observational work should consider additional social tasks besides the three manipulated here and examine the extent to which the tasks exist in a continuous space. Considering laughter makes up, by one estimate, $10 \%$ of vocalizations during conversation (Laskowski \& Burger, 2007), the ability to infer social motivations and predict future social responses using laughter has implications for artificial intelligence, clinical diagnosis, and psychological behavior coding methods. 


\section{Funding}

No funds were used to support this research.

\section{Conflicts of Interest}

On behalf of all authors, the corresponding author states that there is no conflict of interest.

\section{Author Contributions}

A.W. designed, implemented, analyzed, and wrote up this study.

\section{Ethics Approval}

This study was approved by the University of Wisconsin - Madison I.R.B. and was performed to the ethical standards as laid down in the 1964 Declaration of Helsinki.

\section{Data and Code availability}

All study materials, data, audio files, and analysis code and output are available online (https://osf.io/a8t29/).

\section{Acknowledgments}

Jennie Lipson, Tori Lim, Stefan Lizarzaburu, Erika Osherow, Lindsey Porter, London Woodruff, Amelia Boruch, Ty Jager, Caroline Roble, and Solveig Viren collected and coded data. Paula Niedenthal provided feedback. 


\section{References}

Albin, A. L. (2014). PraatR: An architecture for controlling the phonetics software "Praat" with the R programming language. The Journal of the Acoustical Society of America, 135(4), 2198-2199. https://doi.org/10.1121/1.4877175

Andelic, N., Feeney, A., \& McKeown, G. (2019). Evidence for Communicative Compensation in Debt Advice with Reduced Multimodality. 2019 International Conference on Multimodal Interaction, 210-219. https://doi.org/10.1145/3340555.3353757

Anikin, A. (2018). Soundgen: An open-source tool for synthesizing nonverbal vocalizations. Behavior Research Methods. https://doi.org/10.3758/s13428-018-1095-7

Benjamini, Y., \& Hochberg, Y. (1995). Controlling the false discovery rate: A practical and powerful approach to multiple testing. Journal of the Royal Statistical Society. Series B (Methodological), 289-300.

Bryant, G. A., \& Aktipis, C. A. (2014). The animal nature of spontaneous human laughter. Evolution and Human Behavior, 35(4), 327-335.

Bryant, G. A., Fessler, D. M. T., Fusaroli, R., Clint, E., Aarøe, L., Apicella, C. L., Petersen, M. B., Bickham, S. T., Bolyanatz, A., Chavez, B., Smet, D. D., Díaz, C., Fančovičová, J., Fux, M., Giraldo-Perez, P., Hu, A., Kamble, S. V., Kameda, T., Li, N. P., ... Zhou, Y. (2016). Detecting affiliation in colaughter across 24 societies. Proceedings of the National Academy of Sciences, 113(17), 4682-4687. https://doi.org/10.1073/pnas.1524993113

Buuren, S. van, \& Groothuis-Oudshoorn, K. (2011). mice: Multivariate Imputation by Chained Equations in R. Journal of Statistical Software, 45(3), 1-67. 
Cann, A., Zapata, C. L., \& Davis, H. B. (2009). Positive and Negative Styles of Humor in Communication: Evidence for the Importance of Considering Both Styles. Communication Quarterly, 57(4), 452-468. https://doi.org/10.1080/01463370903313398

Champely, S. (2020). pwr: Basic Functions for Power Analysis. https://CRAN.Rproject.org/package $=$ pwr

Cowen, A. S., \& Keltner, D. (2017). Self-report captures 27 distinct categories of emotion bridged by continuous gradients. Proceedings of the National Academy of Sciences, 114(38), E7900-E7909. https://doi.org/10.1073/pnas.1702247114

Curran, W., McKeown, G. J., Rychlowska, M., André, E., Wagner, J., \& Lingenfelser, F. (2018). Social Context Disambiguates the Interpretation of Laughter. Frontiers in Psychology, 8. https://doi.org/10.3389/fpsyg.2017.02342

Decomposing, Probing, and Plotting Interactions in R. (n.d.). UCLA: Statistical Consulting Group. Retrieved August 13, 2020, from https://stats.idre.ucla.edu/r/seminars/interactions-r/

Devereux, P. G., \& Ginsburg, G. P. (2001). Sociality effects on the production of laughter. The Journal of General Psychology, 128(2), 227-240. https://doi.org/10.1080/00221300109598910

Laskowski, K., \& Burger, S. (2007). On the correlation between perceptual and contextual aspects of laughter in meetings. Proc. ICPhS WS on Phonetics of Laughter, 55-60.

Lavan, N., Scott, S. K., \& McGettigan, C. (2015). Laugh like you mean it: Authenticity modulates acoustic, physiological and perceptual properties of laughter. Journal of Nonverbal Behavior, 1-17. 
Mehu, M., \& Dunbar, R. I. M. (2008). Naturalistic observations of smiling and laughter in human group interactions. Behaviour, 145(12), 1747-1780.

Nwokah, E. E., Hsu, H.-C., Davies, P., \& Fogel, A. (1999). The Integration of Laughter and Speech in Vocal Communication: A Dynamic Systems Perspective. Journal of Speech, Language, and Hearing Research, 42(4), 880-894. https://doi.org/10.1044/jslhr.4204.880

Oveis, C., Spectre, A., Smith, P. K., Liu, M. Y., \& Keltner, D. (2016). Laughter conveys status. Journal of Experimental Social Psychology, 65, 109-115.

Owren, M. J., \& Bachorowski, J.-A. (2003). Reconsidering the evolution of nonlinguistic communication: The case of laughter. Journal of Nonverbal Behavior, 27(3), 183-200. https://doi.org/10.1023/A:1025394015198

Rychlowska, M., Jack, R. E., Garrod, O. G. B., Schyns, P. G., Martin, J. D., \& Niedenthal, P. M. (2017). Functional smiles: Tools for love, sympathy, and war. Psychological Science, 0956797617706082. https://doi.org/10.1177/0956797617706082

Scarantino, A. (2018). Emotional Expressions as Speech Act Analogs. Philosophy of Science, 85(5), 1038-1053. https://doi.org/10.1086/699667

Szameitat, D. P., Alter, K., Szameitat, A. J., Darwin, C. J., Wildgruber, D., Dietrich, S., \& Sterr, A. (2009). Differentiation of emotions in laughter at the behavioral level. Emotion (Washington, D.C.), 9(3), 397-405.

Tanaka, H., \& Campbell, N. (2011). Acoustic features of four types of laughter in natural conversational speech. Proceedings of the 17th International Congress of Phonetic Sciences, 1958-1961. 
van de Pol, M., \& Wright, J. (2009). A simple method for distinguishing within- versus betweensubject effects using mixed models. Animal Behaviour, 77(3), 753-758. https://doi.org/10.1016/j.anbehav.2008.11.006

Venables, W. N., \& Ripley, B. D. (2002). Modern Applied Statistics with S (Fourth). Springer. http://www.stats.ox.ac.uk/pub/MASS4

Wood, A., Martin, J., \& Niedenthal, P. (2017). Towards a social functional account of laughter: Acoustic features convey reward, affiliation, and dominance. PLOS ONE, 12(8), e0183811. https://doi.org/10.1371/journal.pone.0183811

Wood, A., \& Niedenthal, P. (2018). Developing a social functional account of laughter. Social and Personality Psychology Compass, 12(4). 